Editorial

\title{
Acknowledgment to Reviewers of Applied Mechanics in 2021
}

\author{
Applied Mechanics Editorial Office
}

MDPI AG, St. Alban-Anlage 66, 4052 Basel, Switzerland

Rigorous peer-reviews are the basis of high-quality academic publishing. Thanks to the great efforts of our reviewers, Applied Mechanics was able to maintain its standards for the high quality of its published papers. Thanks to the contribution of our reviewers, in 2021, the median time to first decision was 21.5 days and the median time to publication was 43 days. The editors would like to extend their gratitude and recognition to the following reviewers for their precious time and dedication, regardless of whether the papers they reviewed were finally published:

\begin{tabular}{|c|c|}
\hline Abdelkrem Eltaggaz & Hannah Stuart \\
\hline Agárdi Anita & Hauke Gravenkamp \\
\hline Albert Patterson & Imre Kiss \\
\hline Albert Zamarin & Jarosław Rybak \\
\hline Aleksander Czekanski & Jaworski C. Capricho \\
\hline Aleksei Vakhnin & Jorge Luis Suzuki \\
\hline Alessandro Mariotti & Jose González \\
\hline Ali Farajpour & Juan David Cano-Moreno \\
\hline Amin Pourasghar & Kevin Bollino \\
\hline Anargyros Karakalas & Kian K. Sepahvand \\
\hline Anna M. Rakoczy & Koji Takahashi \\
\hline Anne Benneker & Krzysztof J. Kurzydłowski \\
\hline Ayman El-Zohairy & Laith Abualigah \\
\hline Ayyappa Atmakuri & Laura Ruiz Cantu \\
\hline Barbara Cosanti & Leon Abelmann \\
\hline Basuraj Bhowmik & Luca Giaccone \\
\hline Bora Pulatsu & Łukasz Sadowski \\
\hline Brahmanandam Javvaji & Madalina Dumitriu \\
\hline Chao Liu & Magdalena Palacz \\
\hline Chenrayan Senthil & Majid Ali \\
\hline Christoph Bode & Marcin Włoch \\
\hline Daniela Sova & Marco Bonopera \\
\hline Eduardo Aoun Tannuri & Marek Szkodo \\
\hline Elena Ferretti & Margarida Quina \\
\hline Elena Pierro & Marina Vukoje \\
\hline Elena Torskaya & Maros Martinkovic \\
\hline Ferenc Markus & Matteo Poggiali \\
\hline Fernando Julian & Mazeyar Parvinzadeh Gashti \\
\hline Francisco Fraga-López & Michael M. Khonsari \\
\hline George E. Varelis & Michał Rogala \\
\hline Giada Rotisciani & Mohamed Hssikou \\
\hline Giuseppe Ruta & Natsuki Tsushima \\
\hline Gyani Shankar Sharma & Noel Peter Tan \\
\hline Hamid Farrokh Ghatte & Ojha Gunendra Prasad \\
\hline
\end{tabular}

Citation: Applied Mechanics Editorial Office. Acknowledgment to Reviewers of Applied Mechanics in 2021. Applied Mechanics 2022, 3 , 121-122. https://doi.org/10.3390/ applmech3010008

Published: 28 January 2022

Publisher's Note: MDPI stays neutral with regard to jurisdictional claims in published maps and institutional affiliations.

Copyright: $\odot 2022$ by the authors. Licensee MDPI, Basel, Switzerland. This article is an open access article distributed under the terms and conditions of the Creative Commons Attribution (CC BY) license (http://creativecommons.org/licenses/by/4.0/).

\author{
Hannah Stuart \\ Hauke Gravenkamp \\ Imre Kiss \\ Jarosław Rybak \\ Jaworski C. Capricho \\ Jorge Luis Suzuki \\ Jose González \\ Juan David Cano-Moreno \\ Kevin Bollino \\ Kian K. Sepahvand \\ Koji Takahashi \\ Laith Abualigah \\ tukasz Sadowski \\ Madalina Dumitriu \\ Magdalena Palacz \\ Majid Ali \\ Marcin Włoch \\ Marco Bonopera \\ Marek Szkodo \\ Margarida Quina \\ Marina Vukoje \\ Maros Martinkovic \\ Matteo Poggial \\ Mazeyar Parvinzadeh Gashti \\ Michael M. Khonsari \\ Michał Rogala \\ Mohamed Hssikou \\ Ojha Gunendra Prasad
}


Oldrich Sucharda

Olga Kudryashova

Olivier Bruls

Paulo Gamboa

Paweł Fritzkowski

Pietro Galizia

Piotr Sielicki

Piotr Szewczyk

Pooria Khalili

Przemysław Strzelecki

Qiang Guo

Radu Muntean

Rohan Chakrabarty

Roman Sturm

Sander Calisal

Sandra Walker

Sebastian Garus

Seong-jin Lee

Sergey N. Shevtsov

Shannon Ryan

Shanqing $\mathrm{Xu}$
Shantonu Biswas

Shengyou Yang

Shipin Qin

Somen K. Bhudolia

Sonia Marfia

Sunil K. Sharma

Supaphorn Thumsorn

Szabolcs Fischer

Tadeusz Chyży

Thomas Heuzé

Tibor Krenicky

Timothy A Sands

Timothy Sands

Van-Vuong Lai

Victor A. Eremeyev

Wei Long Ng

Wu Zhou

Yoji Shibutani

Yong Li

Yoshikazu Kobayashi

Young-Bok Kim 